\title{
Intravaginal TLR agonists increase local vaccine-specific CD8 T cells and human papillomavirus-associated genital-tumor regression in mice
}

\author{
S Domingos-Pereira ${ }^{1,4}$, L Decrausaz $^{1,4}$, L Derré $^{1}, \mathrm{M}_{\text {Bobst }}{ }^{1}$, P Romero $^{2}$, JT Schiller ${ }^{3}$, P Jichlinski ${ }^{1}$ and \\ D Nardelli-Haefliger ${ }^{1}$
}

Human papillomaviruses (HPV)-related cervical cancer is the second leading cause of cancer death in women worldwide. Despite active development, HPV E6/E7 oncogene-specific therapeutic vaccines have had limited clinical efficacy to date. Here, we report that intravaginal (IVAG) instillation of CpG-ODN (TLR9 agonist) or poly-(I:C) (TLR3 agonist) after subcutaneous E7 vaccination increased $\sim$ fivefold the number of vaccine-specific interferon- $\gamma$-secreting CD8 T cells in the genital mucosa (GM) of mice, without affecting the E7-specific systemic response. The IVAG treatment locally increased both E7-specific and total CD8 T cells, but not CD4 T cells. This previously unreported selective recruitment of CD8 T cells from the periphery by IVAG CpG-ODN or poly-(I:C) was mediated by TLR9 and TLR3/ melanoma differentiation-associated gene 5 signaling pathways, respectively. For $\mathrm{CpG}$, this recruitment was associated with a higher proportion of GM-localized CD8 T cells expressing both CCR5 and CXCR3 chemokine receptors and E-selectin ligands. Most interestingly, IVAG CpG-ODN following vaccination led to complete regression of large genital HPV tumors in $75 \%$ of mice, instead of $20 \%$ with vaccination alone. These findings suggest that mucosal application of immunostimulatory molecules might substantially increase the effectiveness of parenterally administered vaccines.

\section{INTRODUCTION}

Cervical cancer, the second leading cause of cancer deaths in women worldwide, is associated to persistent infection of the cervical epithelium by high-risk type human papillomaviruses (HPV), most often type $16 .{ }^{1}$ The recently introduced prophylactic vaccines against specific HPV types are highly effective in preventing persistent infections and precancerous lesions; however, they do not induce regression of established lesions. ${ }^{2}$ As expression of E6 and E7 HPV oncogenes in cervical epithelial cells is required for the maintenance of the cancerous phenotype, they represent attractive target antigens for therapy. Therapeutic HPV vaccines designed to induce specific cytotoxic T-lymphocyte (CTL) responses against tumor cells have shown impressive results in animal models. However, their application in humans has shown modest clinical effectiveness, at best. More than 40 therapeutic vaccination trials (phase I, II, or II/III) against high-risk HPV encompassing a total of 1,830 patients have either been published or are still ongoing (reviewed in ref. 3). To date, no candidate vaccine has demonstrated sufficient efficacy in inducing regression of HPV-induced cervical intraepithelial neoplasia or cancer to warrant commercialization.

In general, persistent HPV infection and cervical intraepithelial neoplasias do not promote inflammation. Therefore, we hypothesized that a limiting factor in the efficacy of therapeutic HPV vaccines to date is the inefficient recruitment of the systemic cell-mediated immune responses they induce to the local mucosal site of the neoplasia. In an attempt to increase targeting of the vaccine-specific immune responses to the genital mucosa (GM), we have here evaluated local application of two

\footnotetext{
${ }^{1}$ Department of Urology, Centre Hospitalier Universitaire Vaudois and University of Lausanne, Lausanne, Switzerland. ${ }^{2}$ Ludwig Center for Cancer Research of the University of Lausanne, Lausanne, Switzerland. ${ }^{3}$ Laboratory of Cellular Oncology, National Cancer Institute, NIH, Bethesda, Maryland, USA. ${ }^{4}$ These authors contributed equally to this work. Correspondence: D Nardelli-Haefliger (dnardell@hospvd.ch) 
TLR agonists (CpG-ODN (CpG, a TLR9 agonist) or poly (I:C) (PIC, a TLR3 agonist)) after parenteral vaccination. In addition to their action as adjuvants, ${ }^{4}$ these TLR ligands are known to modify the expression of selectins, integrins, chemokines, and chemokine receptors, ${ }^{5,6}$ which may affect T-cell migration to effector sites. ${ }^{7}$ Most TLR are expressed by the epithelial cells in the reproductive tract of women ${ }^{8}$ and mice, ${ }^{9}$ and in the latter, local vaginal production of chemokines was shown after intravaginal (IVAG) administration of TLR3 and TLR9 agonists. ${ }^{10}$

We recently showed that parenteral vaccination with an adjuvanted HPV16-E7 polypeptide was able to induce E7-specific CD8 T cells in the GM of mice ${ }^{11}$ and regression of small genital tumors in a novel orthotopic murine model for cervical cancer. ${ }^{12,13}$ However, vaccination alone failed to induce regression of larger genital tumors. In the present study, we examined the possibility that IVAG administration of CpG or PIC following parenteral HPV-16 E7 vaccination could increase local vaccinespecific CD8 T-cell number and improve the rates of genital tumor regression.

\section{RESULTS}

IVAG application of CpG or PIC after a subcutaneous (s.c.) E7 vaccination increased both E7-specific and total CD8 $T$ cells in the cervix-vagina (CV)

We first examined whether IVAG CpG or PIC influences T-cell migration to the CV. Before IVAG treatment, C57BL/6 mice were synchronized in a diestrus-like status to avoid possible variations in the IVAG immunostimulatory activity along the menstrual cycle. Groups of mice were IVAG instilled with phosphatebuffered saline (PBS), CpG, or PIC, and three days later CV cells were analyzed by flow cytometry as depicted in Figure 1a. CD8 T cells in CV were significantly increased after CpG or PIC instillation (mean percentage \pm s.e.m. of $0.31 \pm 0.06, P<0.05$ and $0.46 \pm 0.10, P<0.001$, respectively), as compared with PBS $(0.10 \pm 0.02$, Figure $1 \mathbf{b})$, while CD 4 T cells were not significantly affected $(0.46 \pm 0.05$ and $0.44 \pm 0.11$, respectively) as compared with PBS $(0.27 \pm 0.04$, see Figure 1c). Thus IVAG application of either CpG or PIC mainly resulted in attraction of CD8 T cells in $\mathrm{CV}$.

We then reasoned that to attract E7-specific CD8 T cells in the GM, it was necessary to first induce an E7-specific response by vaccination before IVAG application of $\mathrm{CpG}$ or PIC. Groups of mice were s.c. immunized with a long synthetic E7 peptide together with adjuvants, ${ }^{11}$ and 5 days later PBS (as control), $\mathrm{CpG}$, or PIC was administered IVAG. Mice were killed at day 9, and cells recovered from CV were stained with TetE7 (based on the well-known $\mathrm{H}-2 \mathrm{Db}$-restricted E7 ${ }_{49-57}$ CTL peptide) or TetL1 (based on the irrelevant HPV16 L1 ${ }_{165-173}$ CTL epitope, as negative control) and anti-CD8 antibodies (see Figure 2a, for representative E7-immunized mice IVAG administered with $\mathrm{PBS}, \mathrm{CpG}$, or PIC). The percentage of $\mathrm{TetE} 7^{+} \mathrm{CD} 8^{+}$cells in the $\mathrm{CV}$ of E7-vaccinated mice (Figure $\mathbf{2 b}$ ) that had received IVAG CpG or PIC ( $0.59 \pm 0.11$ and $0.48 \pm 0.09$, respectively) was higher than in those that had received IVAG PBS $(0.07 \pm 0.01, P<0.01$, and $P<0.05$ as compared with CpG and PIC, respectively).
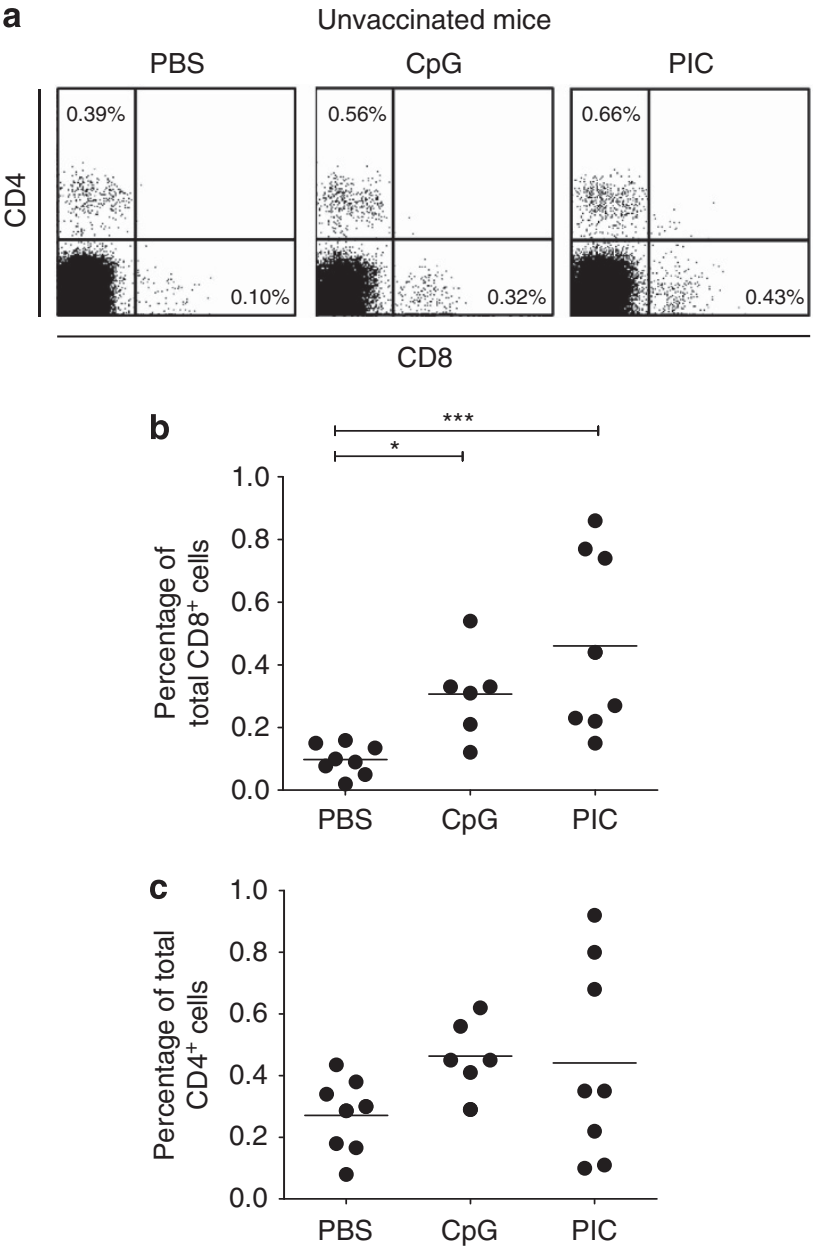

Figure 1 Increase of total CD8 or CD4 T cells in cervix-vagina (CV) upon intravaginal (IVAG) CpG or PIC. Groups of C57BL/6 mice were IVAG instilled with phosphate-buffered saline (PBS), CpG, or PIC, and 3 days later cells recovered from single or pools of two $C V$ were stained with CD8-APC antibodies and CD4-FITC antibodies, and analyzed by flow cytometry. A representative result for each group of mice is shown in (a). Percentage of total CD8 T cells (b) and total CD4 T cells (c) within the recovered CV cells are shown for each IVAG treatment. The horizontal bars represent the mean percentages. Significant differences are indicated by * for $P<0.05$ and ${ }^{* \star *}$ for $P<0.001$.

A similar fivefold increase in total CD8 T cells in CV of the same mice was also observed after the IVAG CpG or PIC treatment (Figure 2c, $3.16 \pm 0.56$ and $2.26 \pm 0.41$ as compared with PBS: $0.43 \pm 0.04, P<0.01$ and $P<0.05$, respectively), which resulted in similar percentages of $\mathrm{TetE} 7^{+} \mathrm{CD} 8{ }^{+}$among the $\mathrm{CD}^{+}$cells (ca. 18\%, Figure 2d) in all mice. This finding, as well as the most unlikely presence of E7 antigen in CV, argues against local proliferation of E7-specific CD8 T cells. In addition and interestingly, the percentage of total CD4 T cells in CV (Figure $2 \mathbf{e}$ and $\mathbf{f}$ ) was not significantly modified by the IVAG CpG or PIC treatments. Together these observations suggest that IVAG CpG or PIC treatment after vaccination preferentially promotes the recruitment of CD8 T cells (and among these, E7-specific CD8 $\mathrm{T}$ cells) from the periphery in $\mathrm{CV}$, while total or $\mathrm{E} 7$-specific CD8 T-cell responses in spleen or genital lymph nodes (GLNs) were 
a

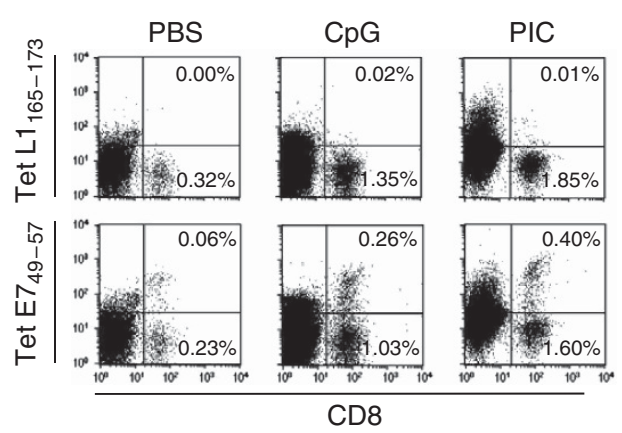

b

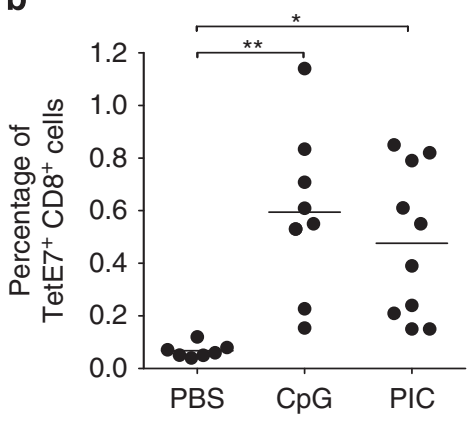

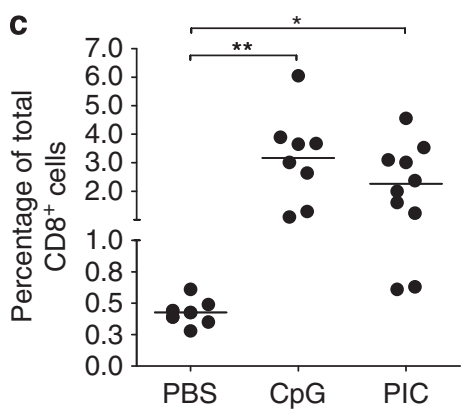

e

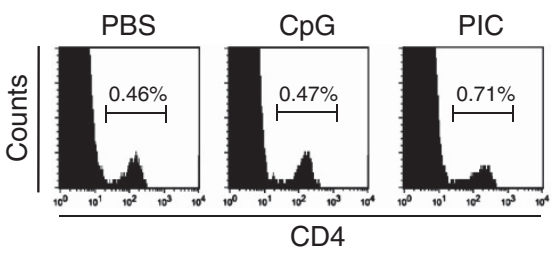

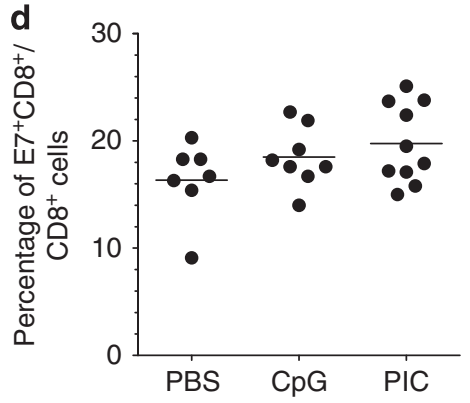

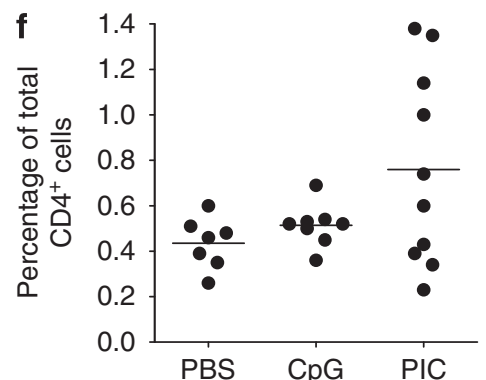

Figure 2 Intravaginal (IVAG) CpG or PIC administered after E7 immunization increased E7-specific and total CD8 T cells in cervix-vagina (CV). Groups of mice were subcutaneously immunized with the adjuvanted E7 vaccine and 5 days later IVAG instilled with phosphate-buffered saline (PBS), $\mathrm{CpG}$, or PIC. Mice were killed 3 days later, at day 9, and cells recovered from pools of two CV were stained with CD8-APC antibodies, CD4-FITC

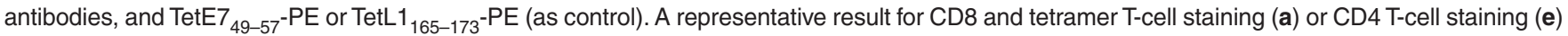
for each group of mice is shown. The percentages of TetE7 ${ }^{+}$CD8 ${ }^{+}$cells (b), total CD8 T cells (c), TetE7 ${ }^{+}$CD8 ${ }^{+}$among the total CD8 T cells (d), and total CD4 T cells (f) within the recovered CV cells are shown for each IVAG treatment. The horizontal bars represent the mean percentages. Significant differences are indicated by ${ }^{\star} P<0.05$ and ${ }^{* *} P<0.01$.

not significantly affected (Table 1). Although CpG and PIC act through different intracellular pathways (discussed below), combining both molecules in IVAG application after vaccination did not have a synergistic effect, as specific and total CD8 $(0.69 \pm 0.11$ and 3.45 \pm 0.66 , respectively) or CD4 T cells $(0.96 \pm 0.20)$ did not significantly differ from those observed after IVAG application of a single TLR agonist.

\section{Increased E7-specific CD8 T-cell responses in CV upon IVAG PIC application after an E7 vaccination is mediated through either TLR3 or Mda5 signaling pathways}

As the particular activity of PIC observed in our experiments was not previously reported, we examined whether it is mediated by the well-documented signaling pathways. PIC is specifically sensed by TLR 3 in the endosome, initiating signaling through the adaptor, Toll/IL-1R domain-containing adaptor inducing interferon (IFN)- $\beta$ (Trif). ${ }^{14}$ In addition, a TLR3-independent cytoplasmic pathway uses melanoma differentiation-associated gene 5 (Mda5), an RNA helicase ${ }^{15}$ that depends exclusively on the CARD adaptor inducing IFN- $\beta$ (Cardif). ${ }^{16}$ We therefore tested PIC as an IVAG immunostimulant after E7 vaccination in Trif knockout (KO), Cardif KO, or Trif-Cardif double-KO mice. For this purpose, groups of $\mathrm{KO}$ mice were immunized with the adjuvanted E7 vaccine and IVAG administered 5 days later with PIC, PBS, or CpG (used in this case as a positive control that should not be affected by the gene deletions). A significantly increased percentage of both $\mathrm{TetE} 7^{+} \mathrm{CD} 8^{+}$cells and total CD8 ${ }^{+}$cells in the CV of IVAG PIC-treated E7-vaccinated Trif KO mice $(0.16 \pm 0.03$ and $0.98 \pm 0.15$, see Figure 3a and $\mathbf{b}$, left panels, respectively) was observed as compared with 
Table 1 Percentage of total TetE7 ${ }^{+} \mathrm{CD}^{+}$and $\mathrm{CD}^{+}$cells in the spleen and GLN of E7-vaccinated mice IVAG challenged with different immunostimulants

\begin{tabular}{|c|c|c|c|c|}
\hline \multirow[b]{2}{*}{ IVAG immunostimulant } & \multicolumn{2}{|c|}{ Spleen } & \multicolumn{2}{|l|}{ GLN } \\
\hline & $\begin{array}{c}\text { Tet E7 }^{+} \text {CD8 }{ }^{+} \\
\text {(mean percentage } \pm \text { s.e.m.) }\end{array}$ & $\begin{array}{c}\text { CD8 }^{+} \text {(mean } \\
\text { percentage } \pm \text { s.e.m.) }\end{array}$ & $\begin{array}{c}\text { Tet E7+ CD8 }{ }^{+} \\
\text {(mean percentage } \pm \text { s.e.m.) }\end{array}$ & $\begin{array}{c}\mathrm{CD}^{+} \text {(mean } \\
\text { percentage } \pm \text { s.e.m.) }\end{array}$ \\
\hline $\mathrm{CpG}$ & $0.74 \pm 0.17(7)$ & $10.18 \pm 0.79(7)$ & $0.21 \pm 0.07(7)$ & $18.04 \pm 0.98(7)$ \\
\hline $\mathrm{PIC}$ & $0.49 \pm 0.06(4)$ & $10.05 \pm 1.22(4)$ & $0.15 \pm 0.02(4)$ & $18.79 \pm 0.44(4)$ \\
\hline
\end{tabular}

Abbreviations: IVAG, intravaginal; GLN, genital lymph node; PBS, phosphate-buffered saline.

aThe number of mice in each group is indicated in parenthesis.

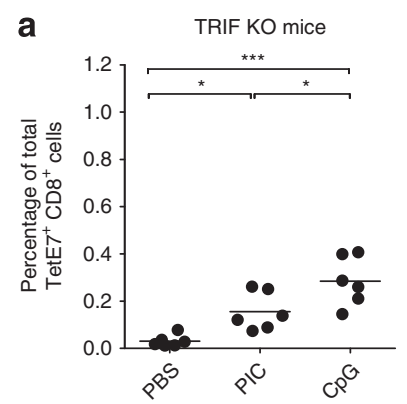

b

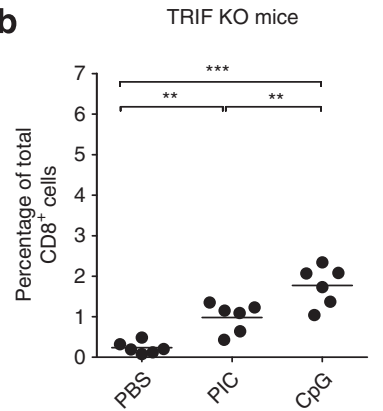

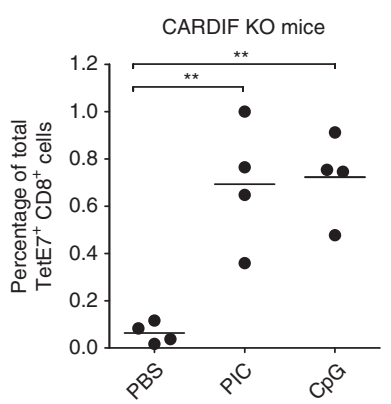

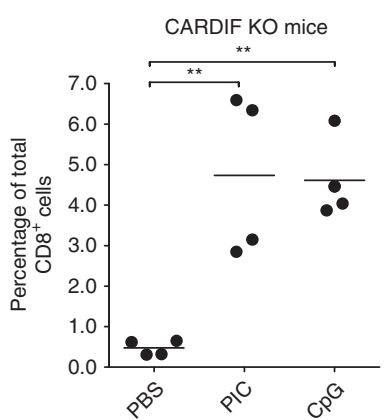

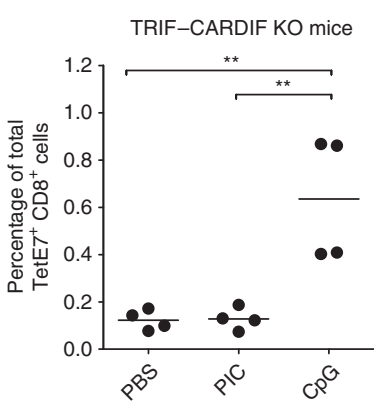

TRIF-CARDIF KO mice

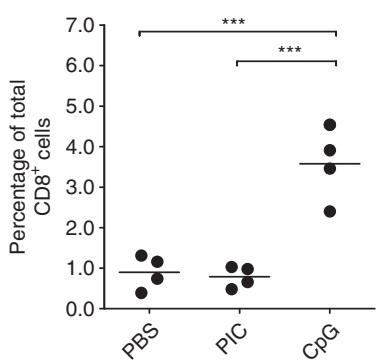

Figure 3 E7-specific and total CD8 T cells in cervix-vagina (CV) of E7-immunized Trif knockout (KO), Cardif KO, and Trif-Cardif double-KO mice intravaginal (IVAG) challenged with CpG of PIC. Groups of Trif KO (left panels), Cardif KO (middle panels), and Trif-Cardif double-KO (right panels) mice were subcutaneously immunized with the adjuvanted E7 vaccine and 5 days later IVAG instilled with phosphate-buffered saline (PBS), PIC, or CpG. Mice were killed three days later, and cells recovered from individual CV were stained with CD8-APC antibodies and TetE7 $49-57^{-P E}$ or TetL $1_{165-173}-\mathrm{PE}$ (as control). The percentages of TetE $7^{+} \mathrm{CD} 8^{+}$cells (a) and total CD8 T cells are indicated (b). The horizontal bars represent the mean percentages. Significant differences are indicated by ${ }^{*} P<0.05,{ }^{* *} P<0.01$, and ${ }^{* * *} P<0.001$.

IVAG PBS-treated mice $(0.03 \pm 0.01, P<0.05$ and $0.24 \pm 0.06$, $P<0.001)$, corresponding to the ca. fivefold increase measured in the wild-type mice (Figure $\mathbf{2 b}$ and $\mathbf{c}$ ). This suggests that PIC may also be recognized by other pathways than TLR3 in the GM. IVAG CpG in these mice induced an even higher increased percentage of both $\mathrm{TetE}^{+} \mathrm{CD} 8^{+}$cells and total $\mathrm{CD} 8^{+}$cells in $\mathrm{CV}(0.29 \pm 0.04$ and $1.77 \pm 0.20$, respectively) as compared with E7-vaccinated Trif KO mice, challenged with PIC $(P<0.05$ and $P<0.01$, respectively). E7-specific and total CD8 T-cell percentages were, however, similar in the spleen of all these mice (see Supplementary Figure S1, left panels) but somewhat lower than those observed in wild-type mice (Table 1), suggesting that Trif signaling might have a modest role in the induction of a systemic $\mathrm{CD} 8+$ response by our vaccine.

Surprisingly, in Cardif $\mathrm{KO}$ mice a significantly increased percentage of both $\mathrm{TetE}^{+}{ }^{+} \mathrm{CD} 8{ }^{+}$cells and total $\mathrm{CD} 8{ }^{+}$cells in
CV was also observed in E7-vaccinated mice upon IVAG PIC treatment $(0.69 \pm 0.13$ and $4.73 \pm 1.00)$ as compared with IVAG PBS $(0.06 \pm 0.02$ and $0.48 \pm 0.09$, respectively, $P<0.01$ for both comparisons, see Figure $\mathbf{3 a}$ and $\mathbf{b}$, middle panels). This ca. 10 -fold increase is also observed when IVAG CpG was given to these mice $(0.72 \pm 0.09$ and $4.61 \pm 0.50$, respectively). This demonstrates that IVAG PIC does not strictly act through Mda5 in the CV of mice. Only in E7-vaccinated Trif-Cardif double-KO mice was IVAG PIC unable to increase the percentages of either E7-specific or total CD8 T cells in their CV $(0.13 \pm 0.02$ and $0.79 \pm 0.13$, respectively) as compared with IVAG PBS-treated mice $(0.12 \pm 0.02$ and $0.90 \pm 0.21$, respectively, see Figure 3a and $\mathbf{b}$, right panels). In contrast, and as expected, IVAG CpG treatment was effective in these mice increasing both TetE7 ${ }^{+}$ $\mathrm{CD} 8{ }^{+}$cells and total CD8 ${ }^{+}$cells in $\mathrm{CV}(0.64 \pm 0.13$ and $3.58 \pm 0.45$, and $P<0.01$ and $P<0.001$, respectively, as compared with either 

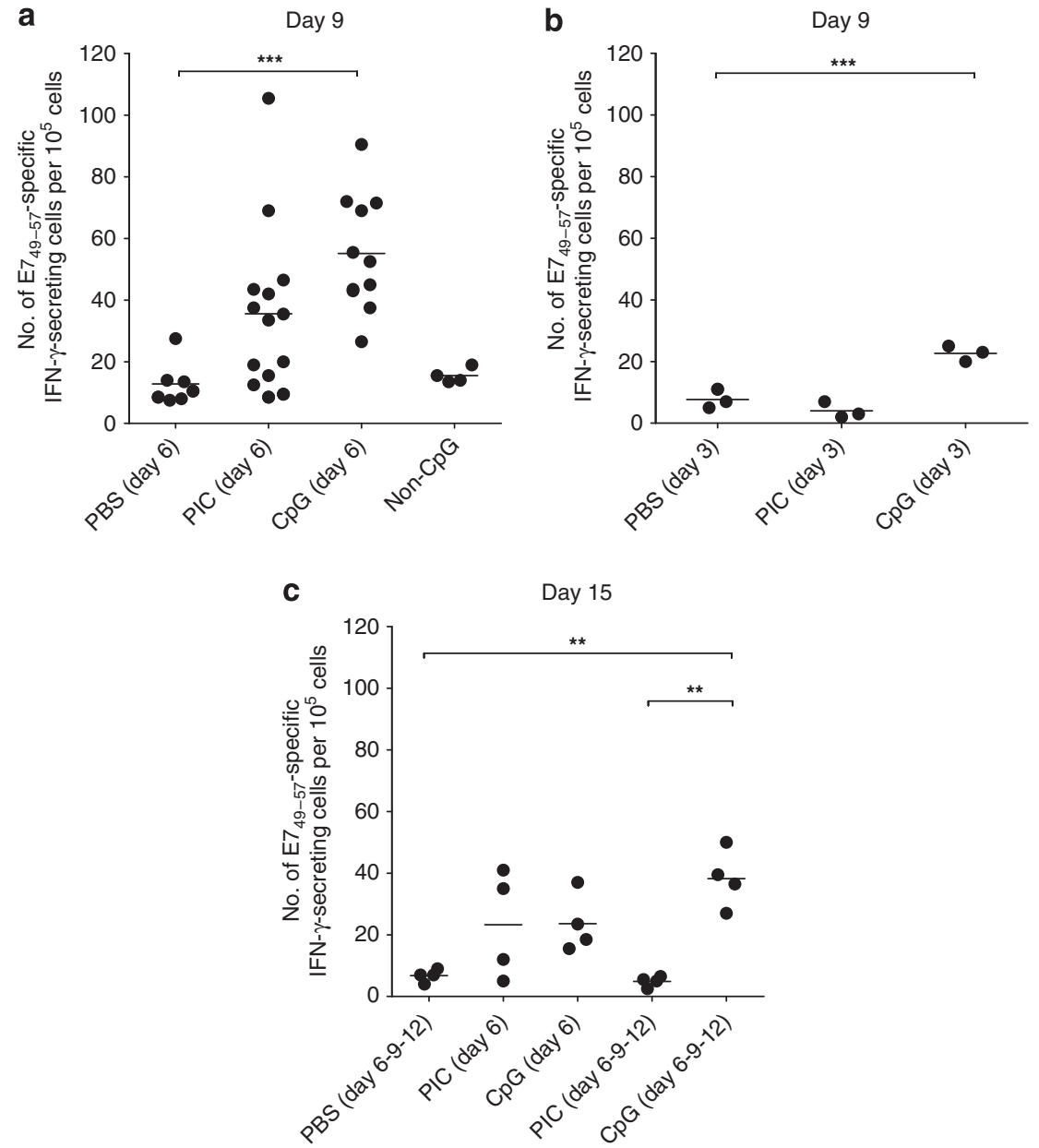

Figure 4 E7-specific interferon- $\gamma($ IFN- $\gamma)$-secreting cells in the genital mucosa after vaccination and intravaginal (IVAG) immunostimulation. Upper panel: mice were subcutaneously (s.c.) immunized with the adjuvanted E7 vaccine at day 1. At day 6 (a) or day 3 (b) phosphate-buffered saline (PBS), PIC, CpG, or Non-CpG were IVAG instilled. Mice were killed at day 9. Lower panel: five groups of mice were s.c. immunized with the adjuvanted E7 vaccine at day 1 . At day 6 , one group was instilled IVAG PBS while two other groups received IVAG PIC and the two last groups had IVAG CpG. A group of each treatment further received IVAG PIC or CpG at days 9 and 12. All mice were killed at day 15. After killing, cells recovered from cervixvagina were analyzed by ex vivo IFN- $\gamma$ ELISPOT. The numbers of $E 7_{49-57}$-specific IFN- $\gamma$-secreting cells per $10^{5}$ cells are indicated for each mouse/ organ. The horizontal bars represent the mean responses. Significant differences are indicated by ${ }^{\star *}$ for $P<0.01$ and ${ }^{* * *}$ for $P<0.001$.

IVAG PBS- or PIC-treated mice). Taken together, these three experiments demonstrate that PIC is sensed by either TLR3 or Mda5 when administered IVAG and, somewhat unexpectedly, that no synergistic effect in CD8 T-cell recruitment seems to be induced when both signaling pathways can be activated.

\section{Successive IVAG CpG applications after E7 vaccination induced increased and sustained E7-specific IFN- $\gamma$-secreting cells in CV}

As CpG is used as adjuvant in our E7 vaccine, it was not possible to use TLR9 KO mice to assess whether preferential recruitment of CD8 T cells after IVAG CpG in our setting was also mediated through the well-described TLR9 signaling pathway. ${ }^{17}$ Instead, we took advantage of control ODNs with the same nucleotide sequence but lacking CpG, which cannot stimulate TLR9, ${ }^{18}$ and examined how their activity would compare with $\mathrm{CpG}$ in our experiments. In addition, we used ex vivo IFN- $\gamma$ ELISPOT assays using the $\mathrm{H}-2 \mathrm{D}^{\mathrm{b}}$-restricted $\mathrm{E} 7{ }_{49-57}$ CTL peptide ${ }^{11}$ to examine whether IVAG CpG or PIC also increased functional E7-specific CD8 T cells and not only tetramer-positive cells. Mice were s.c. immunized with the adjuvanted E7 vaccine and PBS, PIC, CpG or non-CpG control ODN (2138) was IVAG applied 5 days later. At day 9, mice were killed and ex vivo E7-specific IFN- $\gamma$-secreting CD8 T cells were measured in CV (Figure 4a) and peripheral blood mononuclear cells (PBMCs), spleen, and GLN (Supplementary Table S1 online) of individual mice. Our results show that IVAG application of CpG or PIC enhanced the number of E7-specific effector CD8 T cells in the CV (mean E7specific IFN- $\gamma$-secreting CD8 T cells $/ 10^{5}$ cells \pm s.e.m. of $55.2 \pm 5.7$ and 35.6 \pm 7.1 , respectively, as compared with $12.8 \pm 2.6$ for PBS, only significant $P<0.001$ for $\mathrm{CpG}$ ). This increase in E7-specific IFN- $\gamma$-secreting cells in the CV is not higher than the increase of TetE7 ${ }^{+} \mathrm{CD} 8^{+} \mathrm{T}$ cells, thus suggesting that IVAG CpG or PIC does not affect the activation status of the local vaccine-specific CD8 T cells. The ELISPOT data also confirm that the effect of IVAG CpG or PIC is restricted to the CV as E7-specific 
IFN- $\gamma$-secreting CD8 T-cell responses in PBMCs, spleen, and GLN were similar to the group of vaccinated mice treated with IVAG PBS (Supplementary Table S1 online). In addition, E7specific IFN- $\gamma$-secreting cells in CV of mice IVAG treated with non-CpG control ODN (15.5 \pm 1.2$)$ were similar to PBS-treated mice. This suggests that the effect of IVAG CpG does require TLR9 ligation by its cognate agonist.

Given our hypothesis that systemically vaccine-induced CD8 T cells are attracted into the GM upon IVAG application of immunostimulants, we suspected that timing of CpG or PIC application would be important. Indeed, when IVAG CpG or PIC was applied 2 days after E7 vaccination (at a time when vaccine-induced CD8 $\mathrm{T}$ cells cannot be detected ${ }^{19}$ ), E7-specific CD8 T cells measured at day 9 in the $\mathrm{CV}$ of individual mice (see Figure $4 \mathbf{b})$ were similar to PBS for PIC $(7.7 \pm 1.8$ and $4 \pm 1.5$, respectively) and only slightly higher with CpG $(22.7 \pm 1.5$, $P<0.01$, as compared with PBS), but lower than the responses measured with IVAG CpG administered at day 6 (55.2 \pm 5.7 , $P<0.05)$. The duration of the effect of one IVAG administration of CpG or PIC after E7 vaccination was also evaluated beyond day 9 (Figure $4 c$ ) whereby three groups of mice immunized at day 1 with the adjuvanted E7 vaccine, IVAG challenged with PBS, CpG, or PIC at day 6, were killed at day 15 . The E7-specific CD8 T-cell responses in CV tissue after IVAG CpG or PIC were significantly decreased at day $15(23.6 \pm 4.8$ and $23.3 \pm 8.7$, $P<0.01$ for $\mathrm{CpG}$ as compared with day 9 in Figure 4a), though still higher than IVAG PBS (6.8 $\pm 1.0, P=$ not significant). In contrast, three consecutive IVAG CpG doses, but not IVAG PIC, administered at days 6, 9, and 12 (Figure 4c), allowed to sustain the E7-specific CD8 T-cell response measured in CV at day 15 (38.3 \pm 4.7 as compared with $42.0 \pm 2.3$ when measured at day 9 after a single IVAG CpG administration). Besides, this response was higher than that measured at day 15 after PBS, three doses of PIC $(P<0.01$ for both) or one dose of CpG ( $P=$ not significant). This suggests that three consecutive IVAG CpG instillations may, at least transiently, maintain a high E7-specific CD8 $\mathrm{T}$-cell response in $\mathrm{CV}$, while successive PIC applications were not beneficial.

\section{CD8 T cells expressing both CCR5 and CXCR3 chemokine receptors and E-selectin ligands were preferentially increased in CV by IVAG CpG}

To examine the involvement of chemokines and/or endothelial addressins upon IVAG CpG recruitment of CD8 T cell in CV, we mined the data from a recent study from Lindqvist et al. ${ }^{20}$ who provided a genome-wide transcriptome analysis of the effect of IVAG CpG on the mouse genital tract after 4, 24, and $48 \mathrm{~h}$. We reasoned that if certain chemokines and/or endothelial addressins were associated to a preferential recruitment of CD8 $\mathrm{T}$ cells, the respective receptors/ligands should be increased in the array analysis at 24 and/or $48 \mathrm{~h}$. As a control, we first examined how the T-cell coreceptors were modulated by IVAG CpG in the array analysis (see Supplementary Table S2A online). Interestingly, only the $\mathrm{CD} 8 \mathrm{a}$ and $\mathrm{b}$ coreceptors, but not the CD4, were significantly increased by $\mathrm{CpG}$ at 24 and $48 \mathrm{~h}$, in agreement with our findings that CD8 T cells, but not CD4 T cells, were increased by IVAG CpG at $72 \mathrm{~h}$ (Figure 1). We thus extracted from the array analysis a list of all the chemokines and their respective receptors, as well as addressins/selectins and respective ligands/integrins, that were significantly increased by IVAG CpG (see Supplementary Table S2B and C online). To substantiate these observations, the presence of T-cell surface molecules significantly increased at the messenger RNA level 24 and/or $48 \mathrm{~h}$ after IVAG CpG was then analyzed by flow cytometry on the total CD4 and CD8 T cells from the CV of vaccinated mice treated with IVAG PBS or CpG. Computing the percentage of cells within the CD8 or CD4 T-cell populations that expressed these chemokine receptors (Figure 5a) in the presence or absence of IVAG CpG treatment in $\mathrm{CV}$ revealed a significant increase of $\mathrm{CCR}^{+}$and $\mathrm{CXCR} 3^{+}$cells among CD8 T cells recruited upon IVAG CpG (mean percentage \pm s.e.m. of $83.2 \pm 1.4$ and $46.0 \pm 1.2$, respectively, as compared with $70.2 \pm 3.2$ and $35.1 \pm 2.7$, respectively, after IVAG PBS, $P<0.05$ and $P<0.01$, respectively). In sharp contrast, such increases were not observed among CD4 T cells in the IVAG CpG group. Interestingly, it was the double-positive $\mathrm{CCR} 5{ }^{+} \mathrm{CXCR} 3{ }^{+}$cells among the CD8 $\mathrm{T}$ cells that were significantly increased by $\mathrm{CpG}$ $(26.0 \pm 2.1$ to $37.9 \pm 1.1, P<0.05)$ and not CD8 $\mathrm{T}$ cells expressing only one of these chemokine receptors. Moreover, CCR5 ${ }^{+}$ was significantly preferentially expressed by CD8 T cells compared with CD4 T cells after IVAG CpG $(83.2 \pm 1.4$ and $63.9 \pm 1.5$, respectively, $P<0.01)$. Among the integrin/selectin ligands examined (Figure 5b), the percentage of CD8 T cells positive for E-selectin ligand ${ }^{+}\left(\mathrm{ESL}^{+}\right)$was significantly increased by CpG (79.5 \pm 4.9 as compared with PBS 48.0 $\pm 5.8, P<0.01)$, while there was no change in the proportion of $\mathrm{ESL}^{+} \mathrm{CD} 4 \mathrm{~T}$ cells. Interestingly, IVAG CpG treatment was also associated with a significant decreased proportion of $\alpha 4^{+}$integrin (mainly $\alpha 4 \beta 1$, as $\alpha 4 \beta 7$-positive cells were fewer and stable) and of PSGL- $1^{+}$ $\mathrm{CD} 8$, but not $\mathrm{CD} 4, \mathrm{~T}$ cells. In contrast to the $\mathrm{T}$-cell populations in $\mathrm{CV}$, expression of chemokine receptors and integrin/selectin ligands were less affected by IVAG CpG in the T cells from the spleen (Supplementary Table S3 online). In addition to the IVAG CpG-induced modifications of the CD8 T-cell populations in the $\mathrm{CV}$, specific T-cell homing programs to the GM may add a further level of complexity to the outcomes. Indeed, comparison of T-cell populations between spleen, as a surrogate of circulating cells, and CV also shows that irrespective of CpG treatment, $\mathrm{CD} 4 \mathrm{~T}$ cells expressing $\mathrm{CCR} 5^{+}, \mathrm{CXCR}^{+}$, and/or $\mathrm{CCR} 2{ }^{+}$are preferentially recruited to $\mathrm{CV}$, while this is the case only for $\mathrm{CCR}^{+}{ }^{-}$-expressing CD8 T cells. Concerning the integrin/selectin ligands, both CD4 and CD8 T cells expressing $\alpha \mathrm{L}, \mathrm{CD} 43$, ESL, and/or $\alpha 4$ are preferentially recruited to CV. However, significant differences between CD4 and CD8 $\mathrm{T}$ cells can also be noticed as more CD4 T cells expressed ESL (80.9 \pm 2.6 as compared with $48.0 \pm 5.8$ for CD8 T cells, $P<0.01$ ), while more CD8 T cells expressed $\alpha 4$ and PSGL- $1(70.3 \pm 3.0$ and $50.9 \pm 5.0$, respectively, as compared with $43.2 \pm 3.7$ and $20.9 \pm 2.7$, respectively, for $\mathrm{CD} 4 \mathrm{~T}$ cells, $P<0.001$ for both). Altogether, these differences in chemokine receptors and integrin/ligands expression patterns between CD4 and CD8 T cells both upon IVAG CpG and during constitutive homing processes may 

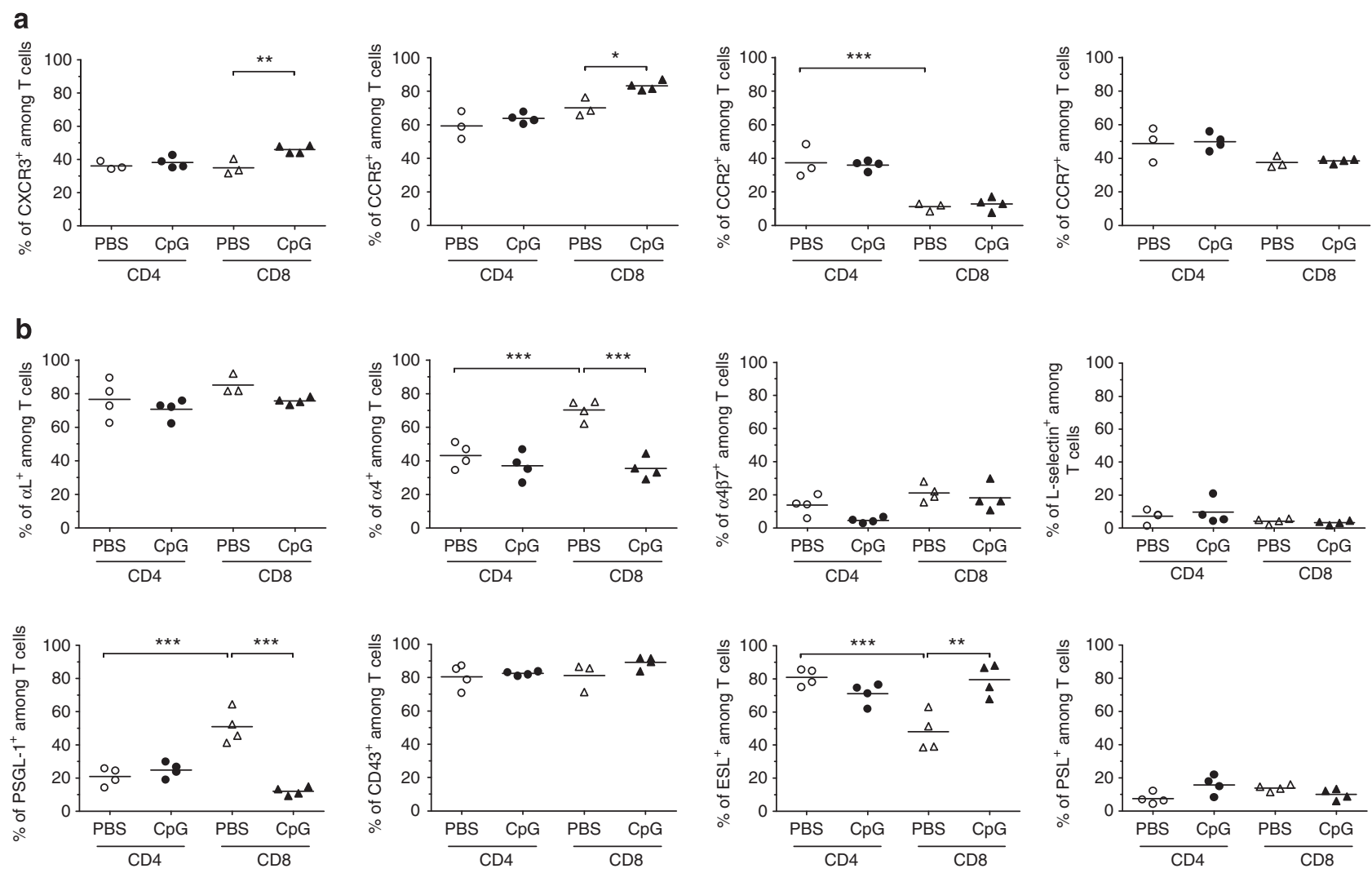

Figure 5 Expression of chemokine receptors and integrin/selectin ligands in the T cells from cervix-vagina (CV) upon intravaginal (IVAG) CpG. Groups of eight mice were subcutaneously immunized with the adjuvanted E7 vaccine, and 5 days later IVAG instilled with phosphate-buffered saline (PBS) (white symbols) or CpG (black symbols). Mice were killed 3 days later, at day 9 , and flow cytometry staining and analysis were performed on cells recovered from pools of two CV. Dead cells were excluded with the aqua dead kit. The percentage of total CD4 (circle) or CD8 (triangle) T cells expressing the chemokine receptors CXCR3, CCR5, CCR2, and CCR7 (a) and integrin/selectin ligands: $\alpha$ L, $\alpha 4, \alpha 4 \beta 7$, L-selectin, PSGL-1, CD43, ESL, and PSL (b) among either total CD4 or CD8 T cells are shown. The horizontal bars represent the mean percentages. Data are representative of results obtained in two independent experiments. Significant differences between IVAG PBS and IVAG CpG treatments within each T-cell populations as well as between the CD4 and CD8 T cells upon IVAG PBS are indicated by ${ }^{*} P<0.05,{ }^{* *} P<0.01$, and ${ }^{* * *} P<0.001$.

account, at least in part, for the preferential recruitment after IVAG CpG of CD8 T cells in CV.

\section{Regression of large genital HPV tumors is enhanced by combining s.c. E7 vaccination with three consecutive IVAG applications of $\mathrm{CpG}$}

We recently developed an orthotopic murine model where HPV16 E6/E7-expressing tumors develop in the genital epithelium after an IVAG instillation of luciferase-expressing TC-1 cells (TC-1-luc). This model more closely mimics the situation of women suffering HPV-induced lesions than previous models involving s.c. injection of tumor cells. ${ }^{12}$ Vaccination of mice harboring small genital HPV tumors with the adjuvanted E7 vaccine induced full tumor regression in $90 \%$ of mice; ${ }^{13}$ however, effective regression was not achieved when genital tumors were larger. We examined here whether combining vaccination with local applications of $\mathrm{CpG}$ could enhance tumor regression, as more E7-specific CD8 T cells would be recruited to CV. For this purpose, six groups of 6-8 mice IVAG challenged with TC-1-luc cells and harboring large tumors at day 12, as shown by monitoring bioluminescence with an in vivo imaging system, were used. Mean bioluminescence of tumors was ca. $1.7 \times 10^{6} \mathrm{p}$ $\mathrm{s}^{-1} \mathrm{~cm}^{-2} \mathrm{sr}^{-1}$ in all groups (see Figure 6a, left panel and one representative group of mice in the right panel) and half of these mice were s.c immunized with the adjuvanted E7 vaccine and the other ones left unvaccinated. One E7-vaccinated group and one control group then received one IVAG dose of CpG 5 days after immunization (at day 17) while two other groups were treated with three consecutive IVAG applications of CpG every 3 days (at days 17, 20, and 23). Percentages of survival upon time (Figure 6b) are shown for each group. Significant extension of survival relative to control untreated mice was only observed in mice receiving E7 vaccination followed by IVAG treatment with either one or three applications of $\mathrm{CpG}(P=0.0036$ and $P=0.0004$, respectively, using adjusted log-rank tests). This was confirmed in independent experiments and by analysis of the compiled data (Figure 6c): 5/10 mice and 9/12 mice were tumor free at 100 days after E7 vaccination followed by one or three IVAG CpG, respectively, as compared with $0 / 13$ untreated mice $(P=0.0075$ and $P=0.0001$, respectively, using Fisher's exact test). Our data further showed that the number of tumor-free mice in the E7-vaccinated and IVAG treated with three CpG 
a

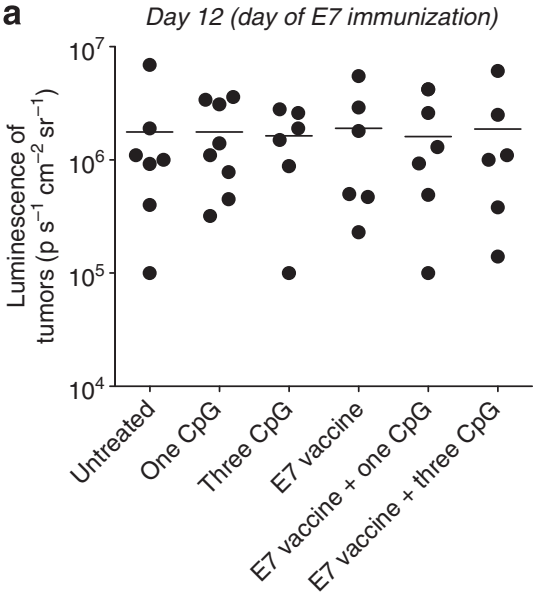

Luminescence of tumors in the « $E 7$ vaccine " group at day 12
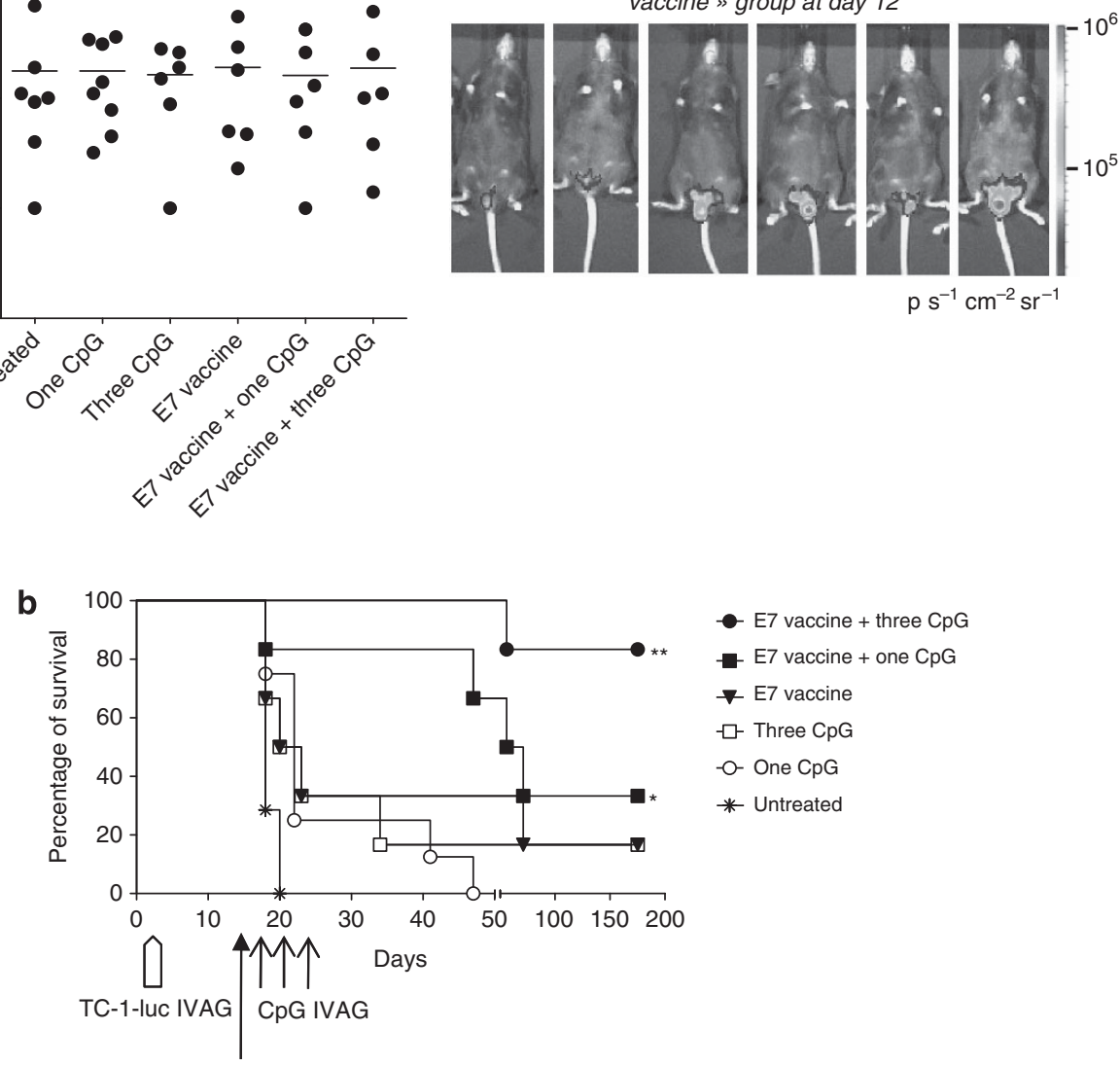

- E7 vaccine + three $\mathrm{CpG}$

E7 vaccine + one CpG

$\checkmark$ E7 vaccine

$-\square$ - Three CpG

-O- One CpG

* Untreated

E7 vaccine s.c.

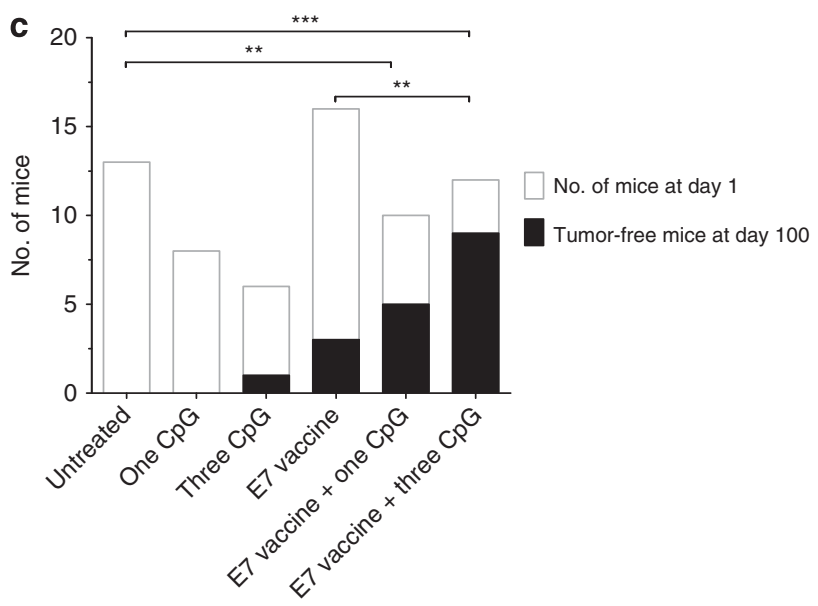

Figure 6 Genital tumor regression upon subcutaneous (s.c.) E7 vaccination followed by intravaginal (IVAG) CpG. Six groups of 6-8 mice were IVAG challenged with TC-1-luc cells at day 1. At day 12, when mice harbored large genital tumors as detected by bioluminescence measurement $\left(\mathrm{ps}^{-1} \mathrm{~cm}^{-2} \mathrm{sr}^{-1}\right)(\mathbf{a})$, three groups were s.c. immunized with the adjuvanted $\mathrm{E} 7$ vaccine and three groups remained unvaccinated. One CpG dose (at day 17) or three CpG doses (at days 17, 20, and 23) were IVAG administered in one E7-vaccinated and one unvaccinated group of mice. Tumor growth/regression was followed twice a week by bioluminescence imaging. Percentage of survival in each group upon time is represented (b). Significant differences following an adjusted log-rank test are indicated by ${ }^{\star} P<0.01,{ }^{\star \star} P<0.002$. Data from independent tumor protection assays were compiled and tumor-free mice at day 100 are shown in (c). Significant differences are indicated by ${ }^{*} P<0.05,{ }^{* *} P<0.01$, and ${ }^{* \star *} P<0.001$ following $\chi^{2}$ tests.

applications group was significantly higher as compared with the only E7-vaccinated mice (9/12 as compared with 3/16 mice, $P=0.0061$ using Fisher's exact test). Interestingly, three IVAG
CpG applications in the absence of E7 vaccination, as well as E7 vaccination alone, also slightly increased survival of mice as compared with untreated mice ( $P=0.0047$ for both, following 


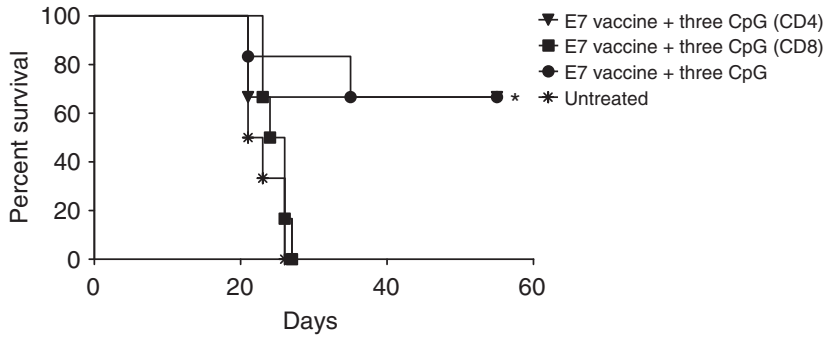

Figure 7 Effect of T-cell depletion on genital tumor regression. Twelve days after TC-1 challenge, mice were either left untreated (stars) or subcutaneous immunized with the adjuvanted E7 vaccine. Four days later, mice received anti-CD4 (triangles), anti-CD8 (squares) antibodies, or left untreated (circles and stars), and treatments were repeated every 6 days. The three vaccinated groups received three doses of CpG that were administrated at days 18,21, and 24. Tumor growth/regression was followed twice a week by bioluminescence imaging. Percentage of survival in each group upon time is represented. Significant differences following an adjusted log-rank test are indicated by ${ }^{\star} P<0.01$.

non-adjusted log-rank tests), though no statistical difference was observed when assessing the number of tumor-free mice at the end of the assay. In fact, ca. 50\% of the mice were already dead before the third IVAG CpG application in the absence of vaccination.

To further examine the involvement of CD8 T cells on the IVAG CpG anti-tumor effect, we determined the effect of antibody-mediated CD8 or CD4 T-cell depletion started $24 \mathrm{~h}$ before the first IVAG CpG application and maintained until day 55 (Figure 7). Vaccination followed by three IVAG CpG applications significantly regressed these large genital tumors $(P=0.0098$, as compared with untreated mice, using adjusted log-rank test), an effect that was significantly diminished by CD8 T-cell depletion $(P=0.0123)$, but left intact by CD4 T-cell depletion. This confirms that CD8 T cells are the mediators of the IVAG CpG antitumor effect. In summary, three consecutive IVAG applications of $\mathrm{CpG}$ represent an additional powerful benefit to E7 vaccination for inducing regression of large genital HPV tumors in mice.

\section{DISCUSSION}

In this study, we demonstrated that IVAG application of $\mathrm{CpG}$ or PIC 5 days after parenteral immunization with a long HPV 16 E7 synthetic peptide and adjuvants in mice led to a major increase of the vaccine-specific CD8 T-cell selectively in the GM. Furthermore, therapeutic E7 vaccination followed by repeated IVAG CpG applications led to the regression of large genital HPV tumors in $75 \%$ of mice, while only $20 \%$ regression was obtained in mice receiving vaccination alone.

Both CpG and PIC are synthetic compounds able to mimic the immune stimulatory activity of microbial DNA and double-stranded RNA, respectively. They were previously shown to be effective adjuvants for protein-based vaccines, mediating maturation of, and cytokine secretion by, dendritic cells (DCs), leading to enhanced antigen presentation, polarization of T-cell responses, and reversal of suppressive regulatory T cell activity. ${ }^{21}$ Topical application of $\mathrm{CpG}$ and/or PIC were also reported to induce cytokine and chemokine secretion leading to attraction of DC and natural killer cells ${ }^{22}$ or macrophages ${ }^{23}$ in mouse lung, $\mathrm{DC}$, and natural killer cells in mouse $\mathrm{GM}^{24}$ or T cells (both CD4 and CD8) in human skin. ${ }^{25}$ To our knowledge, however, this is the first report where preferential attraction of either total or vaccine-specific CD8 T cells over CD4 T cells was induced at the site (in our case, the GM) of CpG or PIC application. Importantly, our data demonstrate that this activity is mediated by the well-described signaling pathways for $\mathrm{CpG}$ and PIC. Indeed, the previously described effects of $\mathrm{CpG}$ involve the direct ligation of TLR $9,{ }^{17}$ while ODNs devoid of the CpG motifs fail to engage TLR9 and deliver the innate response signaling, ${ }^{18}$ as also shown in our experiments (Figure 4a). PIC can signal through two main sensors, i.e., TLR3 in the endosomes ${ }^{14}$ or Mda5, an RNA helicase present in the cytoplasm..${ }^{15}$ Our results in mice deficient for Trif (an adaptor downstream TLR3), Cardif (an adaptor downstream Mda5), or both demonstrated that IVAG PIC after E7 vaccination can attract CD8 T cells into the GM by either one of the two pathways. A third sensor or pathway could be further excluded as neither attraction of E7-specific nor total CD8 T cells could be observed in double-KO Trif-Cardif mice. The redundancy of Trif and Cardif for PIC applied locally is in line with data indicating that the two downstream signaling pathways result in activation of a similar array of transcription factors (i.e., IRF3, JUN, ATF2, and NFKB) leading to the induction of similar genes (reviewed in ref. 26). However, our findings stand in contrast to the required cooperation between the two pathways observed by Kumar et al. ${ }^{27}$ when PIC was used as adjuvant in systemic administration.

As $\mathrm{CpG}$ and PIC act through different TLR signaling pathways (MyD88-dependent (D) and MyD88-independent (I), respectively), one might hypothesize that a synergistic effect could result in higher numbers of attracted total or vaccine-specific CD8 T cells when IVAG CpG and PIC would be administered together. In this regard, Bagchi et al. ${ }^{28}$ showed that D and I agonists (including $\mathrm{CpG}$ and PIC) were markedly synergistic in inducing cytokine production in vivo in mice, while $\mathrm{D}$-specific agonists induced tolerance to one another. However, in contrast to these data, obtained a few hours after systemic use of $\mathrm{CpG}$ and PIC, simultaneous administration of IVAG CpG and PIC did not attract higher numbers of either total or E7-specific CD8 T cells in CV than the single molecules. This difference may be owing to the IVAG route of administration and/or to the different and longer experimental readout in our case. Indeed, the effect of PIC on cytokines/chemokines secretion was shown to only occur at short term and with no additive effect when applying successive doses, in contrast to $\mathrm{CpG}^{29}$ This may also explain the ineffectiveness of successive IVAG PIC doses in our experiments. Chemokines have a key role in innate inflammatory responses leading to recruitment of circulating leukocytes, including $\mathrm{T}$ cells, to sites of inflammation. ${ }^{7}$ In mice, IVAG administration of CpG stimulates a potent response of MIP- $1 \alpha$ (CCL3), MIP$1 \beta$ (CCL4), MIP-2, IP-10 (CXCL10), and RANTES (CCL5) chemokines in the vagina and/or GLN. ${ }^{10}$ However, additional mechanisms by which CpG may contribute to CD8 T-cell attraction to the GM may include induction of adhesion molecules. 
In this regard, $\mathrm{CpG}$ was shown to upregulate ICAM-1 and E-selectin in endothelial cells. ${ }^{30}$ Upon bacterial $^{31}$ or viral ${ }^{32}$ infections, lymphocyte trafficking to the GM may involve both mucosal and non-mucosal homing interactions, and both mucosal adhesion molecule, MadCAM- $1,{ }^{33}$ and non-mucosal adhesion molecules (ICAM-1, VCAM-1, and E-selectin), suggesting that direct induction of these adhesion molecules in the GM by CpG may also occur. Indeed, this was confirmed in a genome-wide transcriptome report ${ }^{20}$ and in our analysis of these data (Supplementary Table $\mathbf{S 2}$ online). Our flow cytometry analysis of chemokine receptors expressed upon IVAG CPG by total T cells in the GM highlighted a population of CD8 T cells that expressed both CCR5 and CXCR3, and thus would be expected to preferentially accumulate in the $\mathrm{CV}$ through interaction with the CpG-induced CCL5, CCL3, CCL4, CXCL9, CXCL10, and/or CXCL11. Upon IVAG CpG treatement, these CD8 T cells also exhibited a combination of high expression of ESL (as determined by direct detection of cell-bound recombinant E-selectin) and low expression of $\alpha 4$ and PSGL-1. ESLs include PSGL-1, but also CD43 (highly expressed by both CD4 and CD8 T cells in CV, Figure 5b) and others, with physiological binding to selectin dependent on the type of glycosylation and/or interactions with other integrins or chemokines. ${ }^{34}$ Interestingly, a combination of both CCR5 and CXCR $3^{35}$ or E-selectin ${ }^{31}$ was also required for pathogen-specific CD4 T-cell recruitment in GM after genital infection by Chlamydia. However, to our knowledge, our study is the first to report on preferential recruitment of total CD8 $\mathrm{T}$ cells over CD4 T cells, as well as on differences in expression of homing molecules between CD4 and CD8 $\mathrm{T}$ cell in the GM or in other mucosae/tissues. Both differential expression of chemokine receptors and of selectin ligands may account for the preferential recruitment of CD8 T cells upon IVAG CpG. However, further investigations will be required to unravel the precise mechanisms involved.

Immunization followed by IVAG CpG to attract systemically induced vaccine-specific CD8 T cells to the tumor site turned out to be an effective strategy for inducing regression of large already established HPV-associated genital tumors in a murine orthotopic model (Figure 7). Our strategy differ from previous cancer immunotherapies, whose rationale for $\mathrm{CpG}$, PIC, or other TLR agonists, beside their use as cancer-vaccine adjuvants, has primarily focused on their ability to increase the antigen-presenting function of $\mathrm{DC},{ }^{36,37}$ thereby directly enhancing tumor-specific CTL responses when used alone ${ }^{38-40}$ or in combination with chemotherapy, ${ }^{41}$ topical skin-bleaching agent, ${ }^{42}$ radiotherapy, ${ }^{43}$ cryosurgery, ${ }^{44}$ or vaccination. ${ }^{45}$ We expect that our strategy may be extended to tumors located in other mucosal sites, as preliminary data (not shown) suggest that intravesical instillation of $\mathrm{CpG}$ after parenteral vaccination similarly results in increased vaccine-specific CD8 T cells in the bladder.

Induction of innate immune responses to counteract the tumor environment was also shown to be especially beneficial in the treatment of tumors located in the GM. This mucosa normally maintains a rather immunosuppressive environment that accommodates commensal bacteria and is permissive for fecundation and implantation of the semi-allogeneic embryo.
This situation favors the development of high-grade lesions and/or cervical cancer after HPV infections, and is correlated with local type II cytokines, absence of IFN- $\gamma$, reduced density and functions of Langerhans cells, ${ }^{46}$ and an increased proportion of regulatory $\mathrm{T}$ cells in the draining $\mathrm{LN}$ in cervical cancer cases. ${ }^{47}$ Interestingly, in the case of vulvar intraepithelial neoplasia, it was recently shown that local long-term treatment with the immune modifier Aldara (a TLR7/8 agonist) before parenteral vaccination was associated with a higher infiltration of effector $\mathrm{T}$ cells in responding lesions. ${ }^{48}$ In our setting, the repetitive application of IVAG CpG alone also slowed genital tumor progression, although tumor-free mice were rarely observed with such aggressively growing tumors. Only the combination of vaccination with IVAG CpG resulted in efficient genital tumor regression.

In conclusion, our study provides an outline for future clinical investigations for eradicating HPV-induced intraepithelial neoplasia or other tumors located in mucosae involving a combination of antigen-specific adaptive immunity followed by the induction of non-specific local innate immunity to promote infiltration of antigen-specific CTL to eradicate the lesions.

\section{METHODS}

Immunization of mice. Eight-week-old female C57BL/6 wild-type mice (Charles River, L'Arbresle, France), Trif KO, Cardif KO, and TrifCardif double-KO mice (kindly provided by Professor J. Tschopp and Aubry Tardivel, Institute of Biochemistry, University of Lausanne, Switzerland (UNIL)) were used following ethical directives of the Swiss veterinary authorities. The $\mathrm{E}_{1-98}, \mathrm{E}_{34-98}$, and E7 ${ }_{49-57}$ peptides ${ }^{11}$ were chemically synthesized by the Protein and Peptide Chemistry Facility of the Institute of Biochemistry (UNIL, Switzerland). Either E7 ${ }_{1-98}$ or E7 ${ }_{34-98}$ (referred thereafter as E7) was used for immunizations as both induced similar E7-specific responses in all organs examined. ${ }^{13}$ Mice were synchronized in a diestrus-like status by a s.c. injection with $0.1 \mu \mathrm{g}$ $\beta$-estradiol and $24 \mathrm{~h}$ later with $2 \mathrm{mg}$ DepoProvera (Pfizer AG, Zurich, Switzerland). The E7 polypeptide $(50 \mu \mathrm{g})$ in combination with the heat-labile enterotoxin HLT ${ }^{49}(0.4 \mu \mathrm{g}$ per dose, Berna-Biotech, Bern, Switzerland) and CpG (\#1826 (5' -TCCATGACGTTCCTGACGTT$3^{\prime}$ ), Coley Pharmaceutical Group, Wellesley, MA, $10 \mu \mathrm{g}$ per dose) were injected s.c. at the base of the tail. PIC (100 $\mu$ g per dose, Sigma, Buchs, Switzerland or Invivogen, Toulouse, France) or CpG (\#1826 or \#2138 (5' -TCCATGAGCTTCCTGAGCTT-3'), Coley Pharmaceutical Group, $100 \mu \mathrm{g}$ per dose) were instilled ( $10 \mu \mathrm{l}$ volume) using a micropipet tip in the vagina of deeply anesthetized mice.

Preparation of PBMCs, splenocytes, GLN, CV cells, and bonemarrow-derived DCs. PBMCs were prepared from tail blood. ${ }^{19}$ Singlecell suspensions of spleen and GLN (ileo-sacral lymph nodes draining the $\mathrm{CV}$ ) were obtained by mechanical dissociation. ${ }^{19}$ The uterine horns were removed from the genital tract, and $\mathrm{CV}$ was minced and digested step wise with $0.5 \mathrm{mg} \mathrm{ml}^{-1}$ thermolysin (Roche, Basel, Switzerland), $1 \mathrm{mg} \mathrm{ml}^{-1}$ collagenase/dispase (Roche), and $2 \mathrm{mg} \mathrm{ml}^{-1}$ DNase (Sigma). ${ }^{19}$ Bone-marrow-derived DCs were generated in the presence of $150 \mathrm{U} \mathrm{ml}^{-1}$ of recombinant mouse granulocyte-macrophage colonystimulating factor (R\&D Systems, Abingdon, UK). ${ }^{13}$

IFN- $\gamma$ ELISPOT assay. IFN- $\gamma$ ELISPOT assay was performed as described in detail in ref. 19. Briefly, 30,000 bone-marrow-derived DCs per well were loaded with $1 \mu \mathrm{g} \mathrm{ml}^{-1}$ of E7 ${ }_{49-57}$ peptide or medium alone (control wells) for $1 \mathrm{~h}$ before adding 100,000 CV cells per well. After 16-24h, E7-specific responses were defined for each individual mouse 
as the number of IFN- $\gamma$ spots per $10^{5}$ cells in the E7-stimulated wells minus the number of IFN- $\gamma$ spots per $10^{5}$ cells in the control wells. The threshold of significance was calculated as the mean +3 s.d. of the E7-specific responses of five naive mice, and corresponded to three spots per $10^{5}$ cells in the spleen and GLN samples and two spots per $10^{5}$ cells in PBMC and CV samples.

Fluorescent tetramer and T-cell labeling. Phycoerythrin (PE)-conjugated E7 ${ }_{49-57}$ and $\mathrm{Ll}_{165-173} \mathrm{H}-2 \mathrm{D}^{\mathrm{b}}$-restricted tetramers (TetE7 and TetL1, respectively, from Ludwig Institute for Cancer Research tetramer production facility, Epalinges, Switzerland) and allophycocyanine (APC)-labeled CD8a (clone 53-6.7, eBioscience, Vienna, Austria) stainings were performed as previously described. ${ }^{11} \mathrm{TetE} 7{ }^{+} \mathrm{CD} 8{ }^{+} \mathrm{T}$ percentages within total cells were calculated after subtraction of the background measured with control TetL1. CD4 labeling was performed using fluorescein isothiocyanate-conjugated anti-CD4 antibodies (clone GK1.5, eBioscience). Cells were analyzed using FACS Calibur and CellQuest Pro software (BD Biosciences, Allschwil, Switzerland). Staining for chemokines and/or integrins/selectins ligands was performed using a 10-color flow cytometry protocol. Stained cells were acquired on a Gallios Flow Cytometer (Beckman Coulter, Nyon, Switzerland) and the multidimensional fluorescence data were analyzed with the FlowJo software (Tree Star, Ashland, OR). The monoclonal anti-mouse antibodies used were: PE/TXRD-anti-CD45/LCA (I3/2.3), PE/TXRD-anti-CD8a (53-6.7), PECy7anti- $\alpha$ L (I21/7) (Southern Biotech, Birmingham, AL); PerCPCy5.5anti-CD3 (17A2), PerCPCy5.5-anti-CD43 (1B11), APC-Cy7-anti-CD8a, AlexaFluor700-anti-CD4 (GK1.5), AlexaFluor488-anti- $\alpha 4$ (R1-2), AlexaFluor488-anti-CCR5 (HM-CCR5), APC-anti- $\alpha 4 \beta 7$ (DATK32), PacificBlue-anti-L-selectin (MEL-14), and PE-Cy7-CXCR3 (CXCR3-173) (Biolegend, London, UK); APC- or PE-Cy7-anti-CD8a (53-6.7), eF450anti-CD4 (GK1.5), and AlexaFluor700-anti-CCR7 (4B12) (eBioscience); PE-anti-PSGL-1 (2PH1, BD Biosciences); and APC-anti-CCR2 (475301) (R\&D Systems). For staining ESL and PSL, cells were incublated with $2 \mu \mathrm{g}$ of E-selectin/human IgG-Fc chimera or $0.1 \mu \mathrm{g}$ of P-selectin/human IgGFc chimera, respectively, (R\&D Systems), in $50 \mu$ l Hank's Balanced Salt Solution (Gibco, Life Technologies, Zug, Switzerland) containing $0.2 \%$ bovine serum albumin (Sigma), before the other extracellular stainings. Selectin chimeras were detected with an AlexaFluor647-anti-human IgG, FC $\gamma$ (Jackson Immunoresearch, Suffolk, UK). Dead cells were stained with a live/dead fixable aqua dead cell stain kit (Invitrogen, Life Technologies).

Challenge of mice with tumor cells. The TC- 1 cell line (C57BL/6 primary lung epithelial cells transduced with retroviral vectors expressing HPV16 E6/E7 and activated c-Ha-ras) was kindly provided by Professor T.-C. Wu (Johns Hopkins Medical Institutions, Baltimore, MD) and further transduced with a luciferase-expressing lentiviral vector to generate TC-1-luc cells. ${ }^{12}$ Anesthetized diestrus synchronized mice were IVAG pretreated with $4 \%$ nonoxynol-9 (N9, Igepal, Sigma) for $6 \mathrm{~h}$, washed with PBS, and IVAG challenged with 20,000 TC-1-luc cells. Genital tumor growth was monitored by bioluminescence $15 \mathrm{~min}$ after an intraperitoneal injection of D-luciferin (Promega, Dübendorf, Switzerland, $150 \mathrm{\mu gg}^{-1}$ of body weight) in a Xenogen imaging system (IVIS lumina, Xenogen/Caliper Life Science, kindly provided by Cellular Imaging Facility, UNIL, Lausanne).

In vivo T-cell depletion. Monoclonal rat anti-CD8 and anti-CD4 IgG antibodies ${ }^{11}$ were purified from GK1.5. and 2.43 rat hybridomas (kindly provided by Dr Martin Kast, University of Southern California, Los Angeles, CA). One hundred microgram of anti-CD8 antibody or $200 \mu \mathrm{g}$ of anti-CD4 antibody were intraperitoneally injected every 6 days to maintain close to $100 \%$ cell depletion in spleen, PBMCs, and GLN as examined by flow cytometry in pilot experiments. Specific cell depletions were further assessed in PBMCs from mice bled during the tumor protection assay.

Statistical analysis. Statistical analyses were performed using Prism 5.00 for Windows (GraphPad software, San Diego, CA). Multiple comparisons were performed using Kruskal-Wallis or one-way analysis of variance followed by a Dunn's or Tukey's post-hoc test, respectively. Other statistical tests are indicated in the text or figure legends.

SUPPLEMENTARY MATERIAL is linked to the online version of the paper at http://www.nature.com/mi

\section{ACKNOWLEDGMENTS}

This work was supported by Oncosuisse (OCS 02304-082008), the Swiss National Science Foundation (\#310000-112406 and 31003A-135109), and the Fondation Emma Muschamp. We thank Dr Roland Sahli for his help in handling the array data and Virginie Martin for her technical help.

\section{DISCLOSURE}

Denise Nardelli-Haefliger is an inventor on patent PCT/IB2009/051372: 'Method and Vaccine for optimizing the specific immune responses'. The remaining authors declared no conflict of interest.

(C) 2013 Society for Mucosal Immunology

\section{REFERENCES}

1. Bosch, F.X., Lorincz, A., Munoz, N., Meijer, C.J. \& Shah, K.V. The causal relation between human papillomavirus and cervical cancer. J. Clin. Pathol. 55, 244-265 (2002).

2. Schiller, J.T., Castellsague, X., Villa, L.L. \& Hildesheim, A. An update of prophylactic human papillomavirus $L 1$ virus-like particle vaccine clinical trial results. Vaccine 26 (Suppl 10), K53-K61 (2008).

3. Gissmann, L. \& Nieto, K. The therapeutic vaccine: is it feasible? Arch. Med. Res. 40, 493-498 (2009).

4. Schwarz, K. et al. Role of Toll-like receptors in costimulating cytotoxic T cell responses. Eur. J. Immunol. 33, 1465-1470 (2003).

5. Matsushima, H., Yamada, N., Matsue, H. \& Shimada, S. TLR3-, TLR7-, and TLR9-mediated production of proinflammatory cytokines and chemokines from murine connective tissue type skin-derived mast cells but not from bone marrow-derived mast cells. J. Immunol. 173, 531-541 (2004).

6. Schaefer, T.M., Fahey, J.V., Wright, J.A. \& Wira, C.R. Innate immunity in the human female reproductive tract: antiviral response of uterine epithelial cells to the TLR3 agonist poly(l:C). J. Immunol. 174, 992-1002 (2005).

7. Charo, I.F. \& Ransohoff, R.M. The many roles of chemokines and chemokine receptors in inflammation. N. Engl. J. Med. 354, 610-621 (2006).

8. Nasu, K. \& Narahara, H. Pattern recognition via the Toll-like receptor system in the human female genital tract. Mediators Inflamm. 2010, 976024 (2010).

9. Soboll, G., Schaefer, T.M. \& Wira, C.R. Effect of Toll-like receptor (TLR) agonists on TLR and microbicide expression in uterine and vaginal tissues of the mouse. Am. J. Reprod. Immunol. 55, 434-446 (2006).

10. Nurkkala, M., Nordstrom, I., Telemo, E. \& Eriksson, K. MHC expression and chemokine production in the murine vagina following intra-vaginal administration of ligands to toll-like receptors 3, 7 and 9. J. Reprod. Immunol. 73, 148-157 (2007).

11. Decrausaz, L. et al. Induction of Human papillomavirus oncogene-specific CD8 T cell effector responses in the genital mucosa of vaccinated mice. Int. J. Cancer 126, 2469-2478 (2010).

12. Decrausaz, L. et al. A novel mucosal orthotopic murine model of Human papillomavrius-associated genital cancers. Int. J. Cancer 128, 2105-2113 (2011).

13. Decrausaz, L. et al. Parenteral but not mucosal immunization is able to induce regression of human papillomavirus associated genital tumors. Int. J. Cancer 129, 762-772 (2011)

14. Yamamoto, M. et al. Role of adaptor TRIF in the MyD88-independent toll-like receptor signaling pathway. Science 301, 640-643 (2003).

15. Kato, H. et al. Differential roles of MDA5 and RIG-I helicases in the recognition of RNA viruses. Nature 441, 101-105 (2006).

16. Meylan, E. et al. Cardif is an adaptor protein in the RIG-I antiviral pathway and is targeted by hepatitis C virus. Nature 437, 1167-1172 (2005).

17. Latz, E., Visintin, A., Espevik, T. \& Golenbock, D.T. Mechanisms of TLR9 activation. J. Endotoxin. Res. 10, 406-412 (2004).

18. Bauer, S. et al. Human TLR9 confers responsiveness to bacterial DNA via species-specific CpG motif recognition. Proc. Natl Acad. Sci. USA 98, 9237-9242 (2001) 
19. Revaz, V., Debonneville, A., Bobst, M. \& Nardelli-Haefliger, D. Monitoring of vaccine-specific gamma interferon inductionin in genital mucosa of mice by real-time reverse-transcription-PCR. Clin. Vacc. Immunol. 5, 757-764 (2008).

20. Lindqvist, M. et al. Unraveling molecular signatures of immunostimulatory adjuvants in the female genital tract through systems biology. PLoS One 6, e20448 (2011).

21. Iwasaki, A. \& Medzhitow, R. Toll-like receptor control of the adaptive immune responses. Nat. Immunol. 10, 987-995 (2004).

22. Pesce, I. et al. Intranasal administration of CpG induces a rapid and transient cytokine response followed by dendritic and natural killer cell activation and recruitment in the mouse lung. J. Innate Immun. 2, 144-159 (2010).

23. Antonelli, L.R. et al. Intranasal poly-IC treatment exacerbates tuberculosis in mice through the pulmonary recruitment of a pathogen-permissive monocyte/macrophage population. J. Clin. Invest. 120, 1674-1682 (2010).

24. Ashkar, A.A., Bauer, S., Mitchell, W.J., Vieira, J. \& Rosenthal, K.L. Local delivery of $\mathrm{CpG}$ oligodeoxynucleotides induces rapid changes in the genital mucosa and inhibits replication, but not entry, of herpes simplex virus type 2. J. Virol. 77, 8948-8956 (2003).

25. Haining, W.N. et al. CpG oligodeoxynucleotides alter lymphocyte and dendritic cell trafficking in humans. Clin. Cancer Res. 14, 5626-5634 (2008).

26. Borden, E.C. et al. Interferons at age 50: past, current and future impact on biomedicine. Nat. Rev. Drug. Discov. 6, 975-990 (2007).

27. Kumar, H., Koyama, S., Ishii, K.J., Kawai, T. \& Akira, S. Cutting edge: cooperation of IPS-1- and TRIF-dependent pathways in poly ICenhanced antibody production and cytotoxic $T$ cell responses. J. Immunol. 180, 683-687 (2008).

28. Bagchi, A. et al. MyD88-dependent and MyD88-independent pathways in synergy, priming, and tolerance between TLR agonists. J. Immunol. 178, 1164-1171 (2007).

29. Herbst-Kralovetz, M.M. \& Pyles, R.B. Quantification of poly(l:C)-mediated protection against genital herpes simplex virus type 2 infection. J. Virol. 80, 9988-9997 (2006).

30. El Kebir, D., Jozsef, L., Pan, W., Wang, L. \& Filep, J.G. Bacterial DNA activates endothelial cells and promotes neutrophil adherence through TLR9 signaling. J. Immunol. 182, 4386-4394 (2009).

31. Kelly, K.A., Chan, A.M., Butch, A. \& Darville, T. Two different homing pathways involving integrin beta7 and E-selectin significantly influence trafficking of CD4 cells to the genital tract following chlamydia muridarum infection. Am. J. Reprod. Immunol. 61, 438-445 (2009).

32. Koelle, D.M., Gonzalez, J.C. \& Johnson, A.S. Homing in on the cellular immune response to HSV-2 in humans. Am. J. Reprod. Immunol. 53, 172-181 (2005).

33. Hawkins, R.A., Rank, R.G. \& Kelly, K.A. Expression of mucosal homing receptor alpha4beta7 is associated with enhanced migration to the Chlamydia-infected murine genital mucosa in vivo. Infect. Immun. 68, 5587-5594 (2000).
34. Zarbock, A., Ley, K., McEver, R.P. \& Hidalgo, A. Leukocyte ligands for endothelial selectins: specialized glycoconjugates that mediate rolling and signaling under flow. Blood 118, 6743-6751 (2011).

35. Olive, A.J., Gondek, D.C. \& Starnbach, M.N. CXCR3 and CCR5 are both required for $\mathrm{T}$ cell-mediated protection against $\mathrm{C}$. trachomatis infection in the murine genital mucosa. Mucosal Immunol. 4, 208-216 (2011).

36. Jakob, T., Walker, P.S., Krieg, A.M., Udey, M.C. \& Vogel, J.C. Activation of cutaneous dendritic cells by CpG-containing oligodeoxynucleotides: a role for dendritic cells in the augmentation of Th1 responses by immunostimulatory DNA. J. Immunol. 161, 3042-3049 (1998).

37. Li, J. et al. Lymphoma immunotherapy with CpG oligodeoxynucleotides requires TLR9 either in the host or in the tumor itself. J. Immunol. 179, 2493-2500 (2007).

38. Heckelsmiller, K. et al. Peritumoral CpG DNA elicits a coordinated response of $\mathrm{CD} 8 \mathrm{~T}$ cells and innate effectors to cure established tumors in a murine colon carcinoma model. J. Immunol. 169, 3892-3899 (2002).

39. Lonsdorf, A.S. et al. Intratumor CpG-oligodeoxynucleotide injection induces protective antitumor T cell immunity. J. Immunol. 171, 3941-3946 (2003).

40. Furumoto, K., Soares, L., Engleman, E.G. \& Merad, M. Induction of potent antitumor immunity by in situ targeting of intratumoral DCs. J. Clin. Invest. 113, 774-783 (2004).

41. Najar, H.M. \& Dutz, J.P. Topical CpG enhances the response of murine malignant melanoma to dacarbazine. J. Invest. Dermatol. 128, 2204-2210 (2008).

42. van den Boorn, J.G. et al. Effective melanoma immunotherapy in mice by the skin-depigmenting agent monobenzone and the adjuvants imiquimod and CpG. PLoS One 5, e10626 (2010).

43. Cerkovnik, P., Jezersek Novakovic, B., Stegel, V. \& Novakovic, S. Class C $\mathrm{CpG}$ oligodeoxynucleotides as a single agent and in combination with radiotherapy efficiently delayed growth of subcutaneous B16F1 tumors. Innate Immunol. 15, 313-321 (2009).

44. Nierkens, S. et al. Route of administration of the TLR9 agonist CpG critically determines the efficacy of cancer immunotherapy in mice. PLoS One 4, e8368 (2009).

45. Geary, S.M., Lemke, C.D., Lubaroff, D.M. \& Salem, A.K. Tumor immunotherapy using adenovirus vaccines in combination with intratumoral doses of CpG ODN. Cancer Immunol., Immunother.: C/l 60, 1309-1317 (2011).

46. Giannini, S.L., Hubert, P., Doyen, J., Boniver, J. \& Delvenne, P. Influence of the mucosal epithelium microenvironment on langerhans cells: implications for the development of squanous intraepithelial lesions of the cervix. Int. J. Cancer 97, 654-659 (2002).

47. Van der Burg, S.H. et al. Association of cervical cancer with the presence of CD4+ regulatory T cells specific for human papillomavirus antigens. Proc. Natl Acad. Sci. USA 104, 12087-12092 (2007).

48. Daayana, S. et al. Phase II trial of imiquimod and HPV therapeutic vaccination in patients with vulval intraepithelial neoplasia. Br. J. Cancer 102, 1129-1136 (2010).

49. Glueck, R. Pre-clinical and clinical investigation of the safety of a novel adjuvant for intranasal immunization. Vaccine 20, S42-S44 (2001). 